women. Surveys in whole populations provide an unusual opportunity to understand the extent and nature of such under-reporting. Methods All consenting individuals aged between 15 and 59 within a demographic surveillance site in northern Malawi were interviewed about their sexual behaviour. Validity of responses was assessed by analysis of probing questions; by comparison of results with in-depth interviews and with Herpes simplex type-2 (HSV-2) seropositivity; by comparing reports to same sex and opposite sex interviewers; and by quantifying the partnerships within the local community reported by men and by women, adjusted for response rates.

Results 6796 women and 5253 men ( $83 \%$ and $72 \%$ of those eligible) consented and took part in sexual behaviour interviews. Probing questions and HSV-2 antibody tests in those who denied sexual activity identified under-reporting for both men and women. Reports varied little by sex or age of the interviewer. The number of marital partnerships reported was comparable for men and women, but men reported about four times as many non-marital partnerships. The discrepancy in reporting of non-marital partnerships was most marked for married women (men reported about seven times as many non-marital partnerships with married women, as were reported by married women themselves), but was only apparent in younger married women see Abstract O2-S3.02 figure 1.

Conclusions We have shown that the under-reporting of non-marital partnerships by women was strongly age-dependent. The extent of under-reporting of sexual activity by young men was surprisingly high. The results emphasise the importance of triangulation, including biomarkers, and the advantages of considering a whole population.

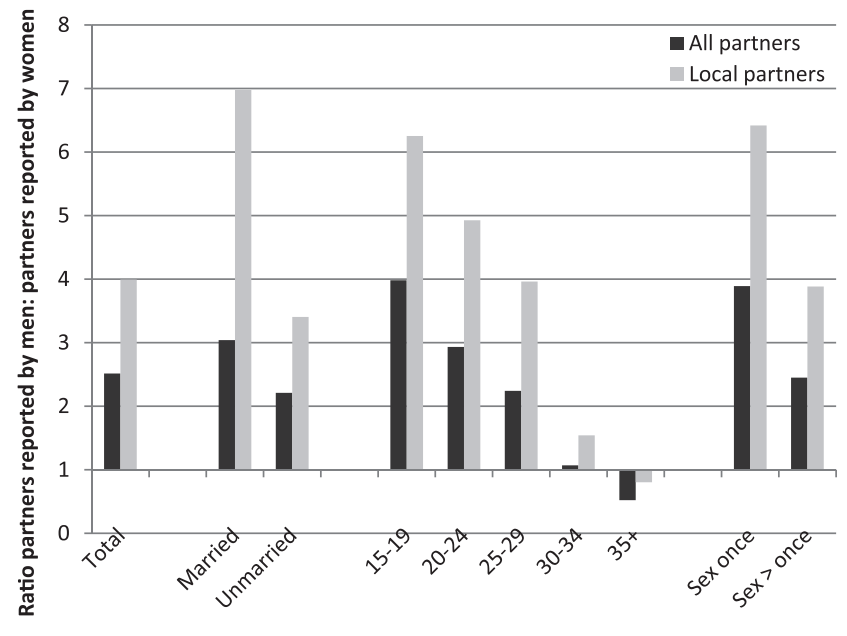

Characteristics of female partner and partnership

Abstract 02-S3.02 Figure 1 Ratio of non-marital partners reported by men compared to women, by characteristics of the women and partnership

\section{2-S3.03 DURATION OF RECENT SEXUAL PARTNERSHIPS IN SEXUALLY ACTIVE MEN AND WOMEN}

doi:10.1136/sextrans-2011-050109.81

Jami Leichliter, Sevgi Aral. Centers for Disease Control \& Prevention, Atlanta, USA

Background The duration of partnerships and partner change rates contribute to STI transmission. Additionally, concurrent partnerships and short gaps between partnerships may be associated with STI spread. Few studies have examined the duration of sexual partnerships at the national level. We examined the duration of recent sexual partnerships reported by sexually active men and women in the US.
Methods Data were from a national survey of reproductive age men and women in the US 7470 men and women reported having an opposite-sex partner in the past year and reported on their recent sex partners (up to three). Duration of partnerships was measured in months; partnerships where respondents reported only having sex once were coded as 1-month in duration. Respondents indicated whether or not the partner was a current sex partner. Bivariate analyses examined duration of partnerships (mean) by demographics. Data were analysed separately for men and women. A subset of unmarried respondents was also examined.

Results Findings were similar for the entire sample and for those who were unmarried. Duration of most recent partnership was longer for women as compared to men, but men reported longer 2 nd and 3rd most recent partnerships than women. Adolescents had the shortest duration for all three partnerships and were least likely to report that the partner was a current sex partner. There was no difference in duration of partnerships by race with the exception of black men having a shorter duration most recent partnership than other men. More black men but fewer black women reported that their most recent partner was a current partner as compared to other groups. However, black and Hispanic men were more likely to indicate that their second recent partnership was also current. For most recent and 2 nd recent partnerships, married men and women reported the longest duration. For most recent partnership, unmarried men and all women with less than a high school education had a longer duration than their counterparts. There were no differences in whether the partner was current by education.

Conclusions Adolescents and black men had the shortest partnerships. Black women did not differ from white or Hispanic women in the duration of their partnerships. Men and women with short partnerships, overlapping and non-overlapping, may play a key role in the transmission of STIs.

\section{2-S3.04 WEB-BASED SEX DIARIES AND YOUNG MEN WHO HAVE SEX WITH MEN: ASSESSING FEASIBILITY, REACTIVITY, AND DATA AGREEMENT}

doi:10.1136/sextrans-2011-050109.82

S Glick, R Winer, M Golden. University of Washington, Seattle, USA

Background Web-based diaries are a promising means for collecting detailed sexual behaviour and substance use data. Few studies have evaluated the feasibility and reliability of collecting these data among men who have sex with men (MSM). We compared quantitative diary data with retrospective survey data collected over 1year in a cohort of young MSM in Seattle, WA.

Methods Ninety-five MSM, age 16-30, completed web-based surveys every 3 months and were randomised to 1 of 4 diary submission schedules: every 2 weeks, once a week, twice a week, or never. The diaries asked questions about daily sexual behaviour and substance use. We assessed the agreement between diary and survey data using $\kappa$ statistics for dichotomous variables and concordance correlation coefficients (CCC) for continuous variables. We used generalised estimating equations to assess for differences in survey data over time between those assigned an active diary schedule to those assigned no diary.

Results During the first 6 months, $78 \%$ of participants assigned to an active diary schedule completed at least $80 \%$ of their diary days, and the 2-week schedule had the highest and most consistent completion rate. Completion rates dropped to $<60 \%$ during the second 6 months. Among participants with active diary schedules, most measures had strong agreement between the diary and survey data (ie, $\kappa$ or $C C C \geq 0.8$ ). The exceptions to this were numbers of overall and unprotected anal sex acts during the first 3 months ( $C C C=0.53$ and 0.57 , respectively) and alcohol use during the first 